\title{
A Direct Attitude Determination Approach Based on GPS Double-Difference Carrier Phase Measurements
}

\author{
Jianhua Cheng, ${ }^{1,2}$ Jing Wang, ${ }^{3}$ and Lin Zhao' \\ ${ }^{1}$ Marine Navigation Research Institute, College of Automation, Harbin Engineering University, Harbin 150001, China \\ ${ }^{2}$ LASSENA Laboratory, Ecole de Technologie Superieure, 1100 Notre-Dame Street West, Montreal, QC, Canada H3C 1K3 \\ ${ }^{3}$ Beihang Univesity, Beijing 100191, China \\ Correspondence should be addressed to Jing Wang; 050441jj@163.com
}

Received 26 November 2013; Accepted 18 February 2014; Published 25 March 2014

Academic Editor: Zheping Yan

Copyright (C) 2014 Jianhua Cheng et al. This is an open access article distributed under the Creative Commons Attribution License, which permits unrestricted use, distribution, and reproduction in any medium, provided the original work is properly cited.

The principle of the traditional attitude solution approach based on GPS (Global Position System) is to get the attitude matrix according to the relationship of coordinates. During the progress, the error of baseline position assumed in ECEF (Earth-Centered Earth-Fixed) and the error of coordinate transform between body frame and reference frame (ENU, East-North-Up) have been included in the result, and finally the precision of attitude determination is reduced. This contribution presents a new approach of attitude determination, in which the attitude angles are calculated by the double-difference carrier phase equation of GPS according to the relationship of attitude matrix and attitude angles through least-squares estimate method. The new approach predigests the procedure of attitude determination which reduces the error assumed. According to the analysis the precision of attitude determination is higher than that of traditional method. It is shown it gets a precise attitude result with the direct attitude determination method in the simulation. A novel algorithm is proposed to solve some problems. Simulation results show the effectiveness of the proposed algorithm.

\section{Introduction}

GPS-based attitude determination is an important research field in recent years, where the key technologies involve the detection and reparation of cycle skip, integer ambiguities resolution, attitude solution, and so forth. At present, the common methods of attitude solution can be divided into two types $[1,2]$.

One type is to get the attitude matrix. Based on the relationship between baseline coordinates in the local orthogonal frame and the body frame, this type includes the TRIAD algorithm which estimates optimally according to the orthogonal property of the attitude matrix, the algorithms based on Wahba problem, for example, QUEST (QUaternion ESTimator), SVD (Singular Value Decomposition), FOAM (Fast Optimal Attitude Matrix), Euler-q, and so forth, and the leastsquare method using multiple epoch baseline coordinate [3]. Besides, resolving the equations which are established by the code or phase observations and the baseline coordinates can also estimate the attitude matrix.
The other type is to get the attitude angles. Setting one baseline along the azimuth axis of vehicle, the yaw and pitch angles can be obtained first, and then according to the rotating relationship of the second baseline, the roll angle can be calculated [4], that is the two-antenna attitude determination method. And the multiple-antenna method is rotating of the other baseline twice and getting the roll angle by the known yaw and pitch angles.

The method of getting attitude matrix includes two steps that estimations of baseline coordinate and attitude matrix parameters, and each of them will lead estimation errors in final results. On the other hand, the attitude matrix derived by the observed equations directly is usually not orthogonal. While, in the method of getting attitude angles the estimations of baseline coordinate and attitude matrix are dropt, and procedure errors are except. But, when the $z$-axis component of all baselines in body frame is zero, the matrix is not full rank that results in unreliable results.

This contribution presents a new approach estimating the attitude angles by double-difference carrier phase equation 
of GPS, which predigests the process calculating attitude angles by attitude matrix. The new method improves the precision and efficiency of estimation and avoids the problem of possible ambiguous values caused by traditional attitudeangle methods.

\section{The Measurement Model of Attitude}

Assume that attitude determination system consists of three antennas, namely, antenna 0 which is set as main antenna and antennas 1 and 2 which are set as slave antennas. All of the three antennas are tracking $M$ satellites. The phase observations collected at receiver $r$ tracking satellite $i$ are modeled as follows:

$$
\varphi_{r}^{i}=\lambda^{-1}\left(r_{r}^{i}-I_{r}^{i}+T_{r}^{i}\right)+f\left(\delta t_{r}-\delta t^{i}\right)+N_{r}^{i}+\varepsilon_{\varphi, r}^{i}
$$

where $\lambda$ and $f$ represent the wavelength and frequency of carrier wave, respectively; $r_{r}^{i}$ is the real range between receiver and satellite $(\mathrm{m}) ; I_{r}^{i}$ is ionospheric effect $(\mathrm{m}) ; T_{r}^{i}$ is tropospheric effect $(\mathrm{m}) ; \delta t_{r}$ is receiver clock error $(\mathrm{s}) ; \delta t^{i}$ is satellite clock error (s); $N_{r}^{i}$ is integer ambiguity; and $\varepsilon_{\varphi, r}^{i}$ is the measurement error.

2.1. Single-Difference Model. Single difference between the two slave receivers can be operated to eliminate many errors, such as the delays of ionospheric and tropospheric, the delays of instrumental and errors of satellite clock. Since the length of baseline is rather less than the distance between receiver and satellite, it is considered that the line-of-sight vectors of two endpoints of baseline are the same [5]. Signal is transmitted as shown in Figure 1.

According to the phase observation equation, when two antennas track the same satellite I, the single-difference equation can be derived as follows:

$$
\varphi_{0 r}^{i}=\lambda^{-1} r_{0 r}^{i}+f \delta t_{0 r}+N_{0 r}^{i}+\varepsilon_{\varphi, 0 r}^{i} .
$$

2.2. Double-Difference Model. Aiming to eliminate receiver clock error the differences between the observations of two satellites taken at the same time and different receivers are formed as follows:

$$
\varphi_{0 r}^{i j}=\varphi_{0 r}^{i}-\varphi_{0 r}^{j}=\lambda^{-1} r_{0 r}^{i j}+N_{0 r}^{i j}+\varepsilon_{\varphi, 0 r}^{i j} .
$$

The purpose of relative positioning is to get the baseline vector $b_{0 r}$. Therefore, it is necessary to contact the doubledifference equation with baseline vector [6]. The line-of-sight vector is $I_{0}^{i}$, and the differences between the distance of main antenna to satellite $i$ and that of slave antenna are $r_{0 r}^{i}$, which is equal to the opposite number of the projection length of baseline vector on $I_{0}^{i}$, it is taken as

$$
r_{0 r}^{i}=-b_{0 r} \cdot I_{0}^{i} .
$$

Therefore, the relationship between double-difference distance and baseline vector can be expressed as

$$
r_{0 r}^{i j}=-b_{0 r} \cdot I_{0}^{i}+b_{0 r} \cdot I_{0}^{j}=-\left(I_{0}^{i}-I_{0}^{j}\right) \cdot b_{0 r} .
$$

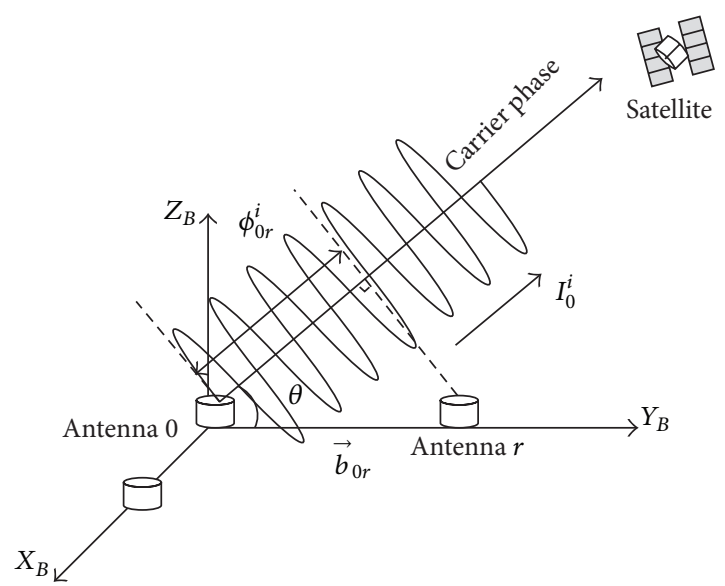

FIGURE 1: Single-difference observation of carrier phase measurements.

The double-difference equation of carrier phase is transformed into

$$
\varphi_{0 r}^{i j}=-\lambda^{-1}\left(I_{0}^{i}-I_{0}^{j}\right) \cdot b_{0 r}+N_{0 r}^{i j}+\varepsilon_{\varphi, 0 r}^{i j}
$$

where the relationship between double-difference $\varphi_{0 r}^{i j}$ and baseline vector $b_{0 r}$ is shown. $\varphi_{0 r}^{i j}$ is double-difference observation, which is a known quantity. $b_{0 r}$ is the baseline vector to be solved. $N_{0 r}^{i j}$ is double-difference integer ambiguity as an unknown quantity.

2.3. The Attitude Determination Model. In traditional process, the attitude determination needs two steps including the estimation of baseline coordinates and attitude matrix.

Aiming to simplify the calculations, avoiding the error during estimation, and improving the precision of solution, a direct method of gaining attitude angles using doubledifference equation is introduced in the following [7].

Assume that GPS attitude determination system is connected rigidly with vehicle; that is, antenna coordinates in body frame are invariant and known which are, respectively, $r_{0, B}, r_{1, B}$, and $r_{2, B}$. The baseline vector $b_{i}$ is equal to $r_{i, B}-r_{0, B}$, where $i$ is equal to 1 or 2 as shown in Figure 2. In the reference frame, there is $r_{i}-r_{0}=R^{T}(\varphi, \theta, \phi) b_{i}$.

According to the transformation relationship of coordinates, baseline vector can be expressed in body frame. Therefore, model (6) is transmitted into

$$
\varphi_{i 0}^{k u}=-\lambda^{-1}\left(I_{0}^{k}-I_{0}^{u}\right) \cdot A_{R}^{E} \cdot R^{T}(\varphi, \theta, \phi) b_{i}+N_{i 0}^{k u}+\varepsilon_{\varphi, i 0}^{k u},
$$

where $R$ is rotation matrix from reference frame to body frame; $A_{R}^{E}$ is transmission matrix from reference frame to ECEF.

After the double-difference integer ambiguity is determined, there are three attitude angles unknown in model (7). Then attitude angles can be estimated by observation equation. 


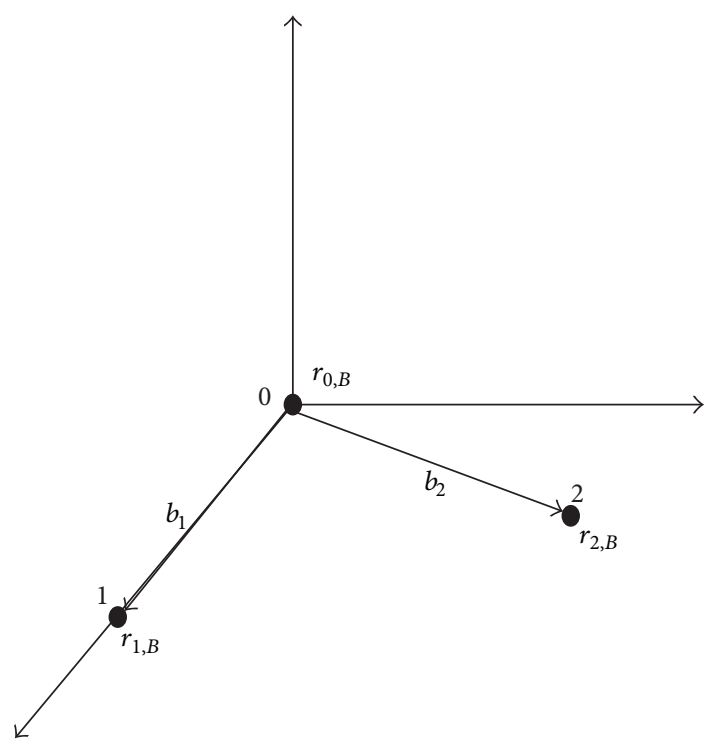

FIGURE 2: The relative position of antenna in the body frame.

M-1 double-difference equations can be established for every baseline:

$$
\begin{aligned}
{\left[\begin{array}{c}
\varphi_{i 0}^{21} \\
\varphi_{i 0}^{31} \\
\vdots \\
\varphi_{i 0}^{M 1}
\end{array}\right]=\lambda^{-1}\left[\begin{array}{c}
-\left(I_{0}^{2}-I_{0}^{1}\right)^{T} \\
-\left(I_{0}^{3}-I_{0}^{1}\right)^{T} \\
\vdots \\
-\left(I_{0}^{M}-I_{0}^{1}\right)^{T}
\end{array}\right] A_{R}^{E} R^{T}(\varphi, \theta, \phi) b_{i} } \\
+\left[\begin{array}{c}
N_{i 0}^{21} \\
N_{i 0}^{31} \\
\vdots \\
N_{i 0}^{M 1}
\end{array}\right],
\end{aligned}
$$

where satellite 1 is the reference satellite. According to relationship between attitude angles and the equation, linearization is operated. Then the final attitude angles can be gained through least-square estimation.

\section{The Nonlinear Least-Square Solution}

The form of rotation matrix $R$ is as follows:

$$
R=\left(\begin{array}{ccc}
C r C y-S r S p S y & C r S y+S r S p C y & -S r C p \\
-C p S y & C p C y & S p \\
S r C y+C r S p S y & S r S y-C r S p C y & C r C p
\end{array}\right),
$$

where $S$ represents sin; $C$ represents cos; $y$ is the yaw angle of vehicle rotating around the $z$-axis of local frame; $r$ is the roll angle of vehicle rotating around the $y$-axis of local frame; and $p$ is the pitch angle of vehicle rotating around $x$-axis of local frame.
Because of the nonlinear relation between the model and attitude angle, attitude angle is determined by nonlinear leastsquare estimation. Assuming that state variables are three attitude angles as follows:

$$
x=\left(\begin{array}{l}
y \\
r \\
p
\end{array}\right) .
$$

Firstly, with the high-order terms being neglected, the nonlinear equation is linearized through first-order Taylor expansion of every equation in the model. The nonlinear equations can be approximately transformed into linear equations expressed by the following matrix:

$$
H \cdot \Delta x=\Delta y .
$$

Observation matrix $H$ can be expressed as follows:

$$
H=\left(\begin{array}{c}
\vdots \\
\cdots-\left(I_{0}^{i}-I_{0}^{1}\right)^{T} A_{R}^{E}\left(\frac{\partial R^{T}}{\partial x_{l}}\right) b_{i} \cdots \\
\vdots
\end{array}\right)
$$

where $x_{l}(l=1,2,3)$ is the attitude angle; $\partial R^{T} / \partial x_{l}$ is attitude matrix's partial derivative to each attitude, which can be expressed as follows:

$$
\begin{aligned}
& \frac{\partial R^{T}}{\partial y}=\left(\begin{array}{ccc}
-C r S y-C y S p S r & -C y C p & -S r S y+C y S p C r \\
C y C r-S y S p S r & -S y C p & C y S r+S y S p C r \\
0 & 0 & 0
\end{array}\right), \\
& \frac{\partial R^{T}}{\partial r}=\left(\begin{array}{ccc}
-C y S r-S y S p C r & 0 & C y C r-S y S p S r \\
-S y S r+C y S p C r & 0 & S y C r+C y S p S r \\
-C p C r & 0 & -C p S r
\end{array}\right), \\
& \frac{\partial R^{T}}{\partial p}=\left(\begin{array}{ccc}
-S y C p S r & S y S p & S y C p C r \\
C y C p S r & -C y S p & -C y C p C r \\
S p S r & C p & -S p C r
\end{array}\right) .
\end{aligned}
$$

$\Delta y=\varphi_{i 0}^{m 1}-f\left(x_{k}\right)$, that is, the deviation of estimation state vector $x_{k}$ to measured value $\varphi_{i 0}^{m 1}$.

Then the least-square solution is

$$
\Delta x=\left(H^{T} H\right)^{-1} H^{T} \Delta y .
$$

According to the equation of nonlinear least-square estimation and the initial attitude angle given above, the estimation of unknown parameters can be obtained. Namely, the attitude angle is estimated by double-difference observation equation of carrier phase.

\section{Error Analysis}

Compared with traditional approach of attitude determination, the solution error of model is analyzed. 
4.1. Error of Traditional Attitude Determination Approach. Assume that the result is obtained from double-difference equation of carrier phase in both methods. In traditional attitude determination method, the baseline coordinates in local frame can be calculated through the equation firstly.

The phase observation error of all satellites is assumed as $\sigma_{\varphi}$, which is independent and its average value is zero. So the error of double-difference observation is $2 \sigma_{\varphi}$. Uniting the double-difference equation of all visible satellites, solution is obtained through least-square estimation in overdetermined equation whose general expression is as follows:

$$
A x=b .
$$
follows:

There is only one least-square solution expressed as

$$
A^{H} A x=A^{H} b \Longrightarrow x=\left(A^{H} A\right)^{-1} A^{H} b .
$$

In (15), error of $b$ is $\delta b$, and error of $A$ is $\delta A$. The effects of $\delta b$ and $\delta A$ on solution $x$ of the equation are proportional to the square of condition number of $A$, that means condition number of overdetermined equation will increase with a quadric function as the following formula shows:

$$
\text { cond }\left(A^{H} A\right)=[\operatorname{cond}(A)]^{2} \text {. }
$$

According to the rule of error transmitting, the error of least-square solution is $4 \sigma^{2}$. Here only the estimation of baseline coordinates in the local frame is finished. More resolution of baseline coordinates are needed in more observed epochs. Then the 9 parameters of three-dimensional attitude matrix can be estimated according to the transformation relationship between baseline coordinates in the body frame and local frame. Unit multiepoch baseline coordinates are as follows:

$$
\left[\begin{array}{c}
X^{B F S} \\
Y^{B F S} \\
Z^{B F S}
\end{array}\right]=R(r)_{Y} R(p)_{X} R(y)_{Z}\left[\begin{array}{c}
X^{L L S} \\
Y^{L L S} \\
Z^{L L S}
\end{array}\right],
$$

where $\left(X^{B F S}, Y^{B F S}, Z^{B F S}\right)$ are the baseline coordinates in the body frame; $\left(X^{L L S}, Y^{L L S}, Z^{L L S}\right)$ are the baseline coordinates in the local frame.

So another least-square estimation is needed to solve the overdetermined equation. Then the estimation error increases to $16 \sigma^{4}$ with a quadric function.

Many different theories are used to solve attitude matrix in the other methods, but the baseline coordinates in the local frame should be estimated at first. And the estimation error also exists in the process of attitude matrix determination. So the error of traditional attitude determination approach is in a high level.

\subsection{Error of the Direct Attitude Determination Approach} Based on Double-Difference Equation. Due to only one step of least-square estimation being operated in the direct attitude determination approach, the error of state vector estimated is as follows:

$$
\begin{aligned}
\operatorname{cov}[\Delta x] & =E\left[\Delta x \Delta x^{T}\right] \\
& =\left(H^{T} H\right)^{-1} H^{T} E\left[\Delta y \Delta y^{T}\right] H\left(H^{T} H\right)^{-1} .
\end{aligned}
$$

Observation errors are mutually independent, whose variance is $4 \sigma^{2}$ and their average is zero. Therefore, $E\left[\Delta y \Delta y^{T}\right]=4 I \sigma^{2}$, where $I$ is unit matrix.

Dimensionless matrix $\widehat{H}$ is defined as $H / b_{0}$, then

$$
\operatorname{cov}[\Delta x]=\left(\widehat{H}^{T} \widehat{H}\right)^{-1}\left(\frac{4 \sigma_{\varphi}^{2}}{b_{0}^{2}}\right) .
$$

Therefore, the error of attitude angle estimated is concerned with the measurement error, measurement matrix, and length of baseline. Ignoring other errors, the longer the baseline is, the higher the precision is; according (20), measurement matrix is only concerned with the geometric position of satellite. The better the geometric distribution is, the less the effect of measurement error on attitude is.

\section{Simulation}

5.1. Designation of Simulation Approach. Through setting the geodetic coordinate of main antenna, the body coordinate of baseline, and the true attitude angles, attitude determination algorithm can be verified by simulation system with the known satellite positions.

Simulation system consists of the following sections.

5.1.1. Obtainment of Satellites' Coordinates in ECEF. With the almanac files supplied by the website of navcen.uscg.gov and actually broadcasted by GPS satellites, the real-time coordinate of all satellites in orbit are calculated; then the coarse precision satellites' tracks can be obtained. After all elevations are calculated in the setting user position, visible satellites information can be obtained.

5.1.2. Simulation of Carrier Signal. After positions of main antenna and reference antenna are set, coordinates of all antennas in the local frame can be obtained according to the predetermined attitude angles. Namely, distance from satellite to antenna is obtained. With certain measurement noise added, carrier signal can be simulated. And the equation of phase observation can be established (assuming that integer ambiguity is determined).

5.1.3. Verification of Attitude Results. Parameters of simulation are known information, and according to different attitude solution approaches, equation is built to solve the attitude angles which will be compared with predetermined attitude angles to verify the algorithm.

5.2. Establishment of Attitude Simulation Model. Installing the array of the three antennas as Figure 3, the system determines attitude of vehicle.

Assume that reference antenna is at the rotation center of vehicle, phase error is set at $(4,5,6) \mathrm{mm}$, and the other simulation conditions are set as Table 1 shown.

Elevation angle limited is set at $5^{\circ}$ [8]; there are 8 visible satellites numbered as Table 2 shows, of which satellite number 22 is the reference satellite. 


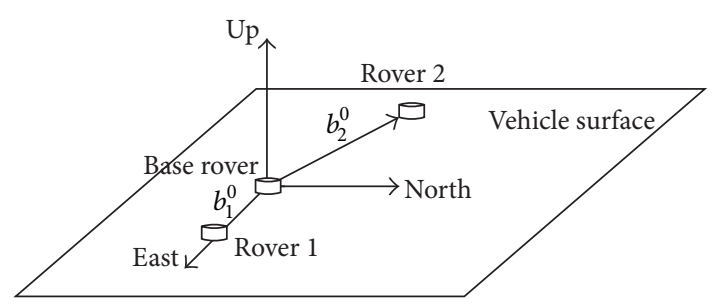

FIGURE 3: The distribution of antenna in the simulation model.

TABLE 1: Settings of simulation parameter.

\begin{tabular}{lc}
\hline Parameter & Value \\
\hline $\begin{array}{l}\text { Reference antenna coordinates in } \\
\text { geographic frame }\end{array}$ & $45^{\circ} \mathrm{N}, 120^{\circ} \mathrm{E}, 0 \mathrm{~m}$ \\
Antenna 1 coordinates in the body frame & $(3,0,0) \mathrm{m}$ \\
Antenna 2 coordinates in the body frame & $(0,3,4) \mathrm{m}$ \\
$\begin{array}{l}\text { Predetermined attitude angles of } \\
\text { simulation }\end{array}$ & Yaw: $90^{\circ}$, roll: $30^{\circ}$, \\
pitch: $30^{\circ}$
\end{tabular}

TABLE 2: The visible satellites in the simulation position.

\begin{tabular}{lcccccccc}
\hline Sequence number & 1 & 2 & 3 & 4 & 5 & 6 & 7 & 8 \\
\hline The visible satellite number & 4 & 14 & 18 & 20 & 22 & 24 & 25 & 29 \\
\hline
\end{tabular}

5.3. Verification of Attitude Results. Because that attitude solution of traditional approach needs multiepochs information, simulation is done for 10 minutes. Namely, 20 epochs is sampled. At the same time, the new approach is simulated. The two attitude determination results are compared.

In traditional attitude determination approach, the results estimated by least-square method once are listed as yaw is $90.0404^{\circ}$, roll is $30.0088^{\circ}$, and pitch is $29.9516^{\circ}$.

Attitude results estimated by nonlinear least-square iteration in the direct double-difference approach are listed as: yaw is $90.1092^{\circ}$, roll is $29.9925^{\circ}$, and pitch is $29.9831^{\circ}$. The estimation process is shown at Figure 4.

According to the result of direct approach, attitude angles become steady after 5 times cyclic calculations within only single epoch. While traditional method need more epochs for obtaining the final attitude solution. Precision of estimation is $10^{-1}\left({ }^{\circ}\right)$ that is higher than the result using traditional attitude determination approach.

\section{Conclusions}

The approach of solving attitude angles based on doubledifference carrier phase measurements is proposed in this contribution, which is analyzed and compared with traditional approach, and some estimation procedures are avoided. The accuracy and low error in calculation of the new attitude determination model are verified. According to the attitude verification program based on MATLAB simulation, reality and reliability are certified and a certain attitude solution precision is achieved.
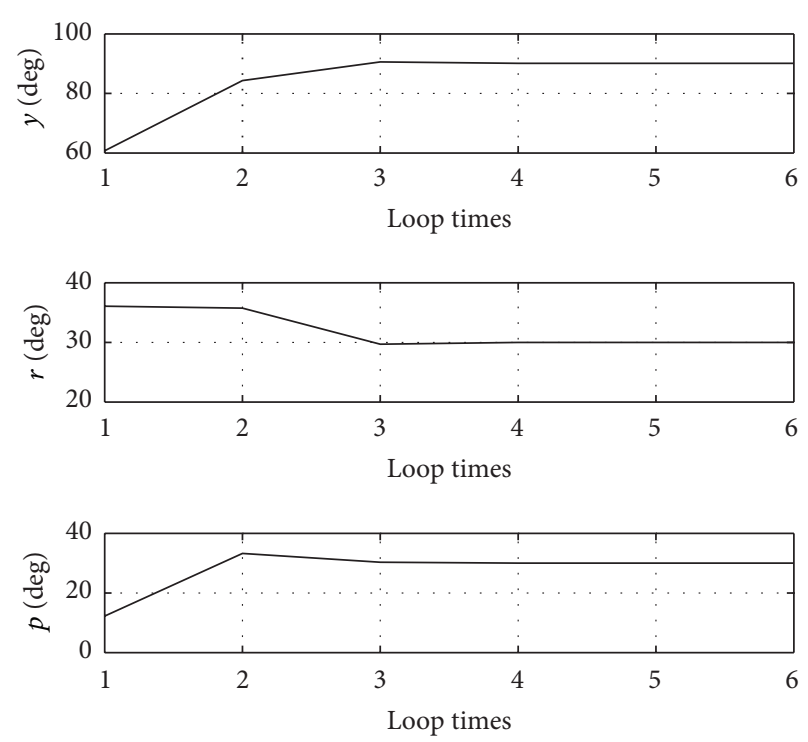

FIGURE 4: The process of attitude angles estimated using the direct approach based on carrier double-difference equation.

\section{Conflict of Interests}

The authors declare there is no conflict of interests regarding the publication of this paper.

\section{Acknowledgments}

Funding for this work was provided by the National Nature Science Foundation of China under Grant no. 61374007 and no. 61104036 . The authors would like to thank all the editors and anonymous reviewers for improving this paper.

\section{References}

[1] P. J. G. Teunissen, G. Giorgi, and P. J. Buist, “Testing of a new single-frequency GNSS carrier phase attitude determination method: land, ship and aircraft experiments," GPS Solutions, vol. 15, no. 1, pp. 15-28, 2011.

[2] G. Giorgi, T. P. Gourlay, P. J. G. Teunissen, L. Huisman, and K. Klaka, "Carrier phase ambiguity resolution for ship attitude determination and dynamic draught," in Proceedings of the 24th FIG International Congress, pp. 11-16, Sydney, Australia, 2010.

[3] J. C. Juang and G.-S. Huang, "Development of GPS-based attitude determination algorithms," IEEE Transactions on Aerospace and Electronic Systems, vol. 33, no. 3, pp. 968-976, 1997.

[4] G. Giorgi, P. J. G. Teunissen, S. Verhagen, and P. J. Buist, “Testing a new multivariate GNSS carrier phase attitude determination method for remote sensing platforms," Advances in Space Research, vol. 46, no. 2, pp. 118-129, 2010.

[5] S. Yoon and J. B. Lundberg, "An integer ambiguity resolution algorithm for real-time GPS attitude determination," Applied Mathematics and Computation, vol. 129, no. 1, pp. 21-41, 2002.

[6] G. Xie, Principles of GPS and Receiver Design, Publishing House of Electronics Industry, Beijing, China, 2011. 
[7] P. J. G. Teunissen, "A canonical theory for short GPS baselinespart I: the baseline precision," Journal of Geodesy, vol. 71, no. 6, pp. 320-336, 1997.

[8] J. Liu, The Principle and Method of GPS Navigation, Publication of Science, Beijing, China, 2008. 


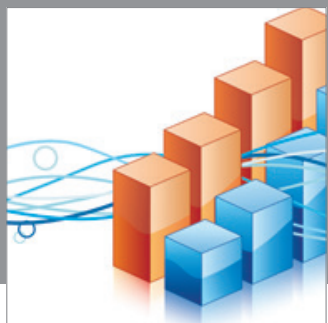

Advances in

Operations Research

mansans

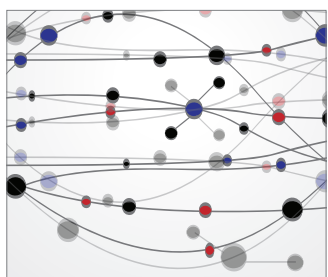

The Scientific World Journal
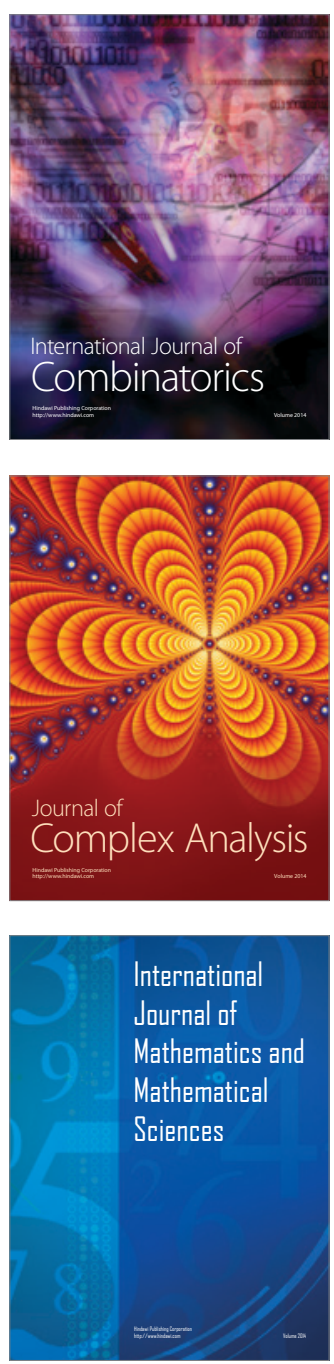
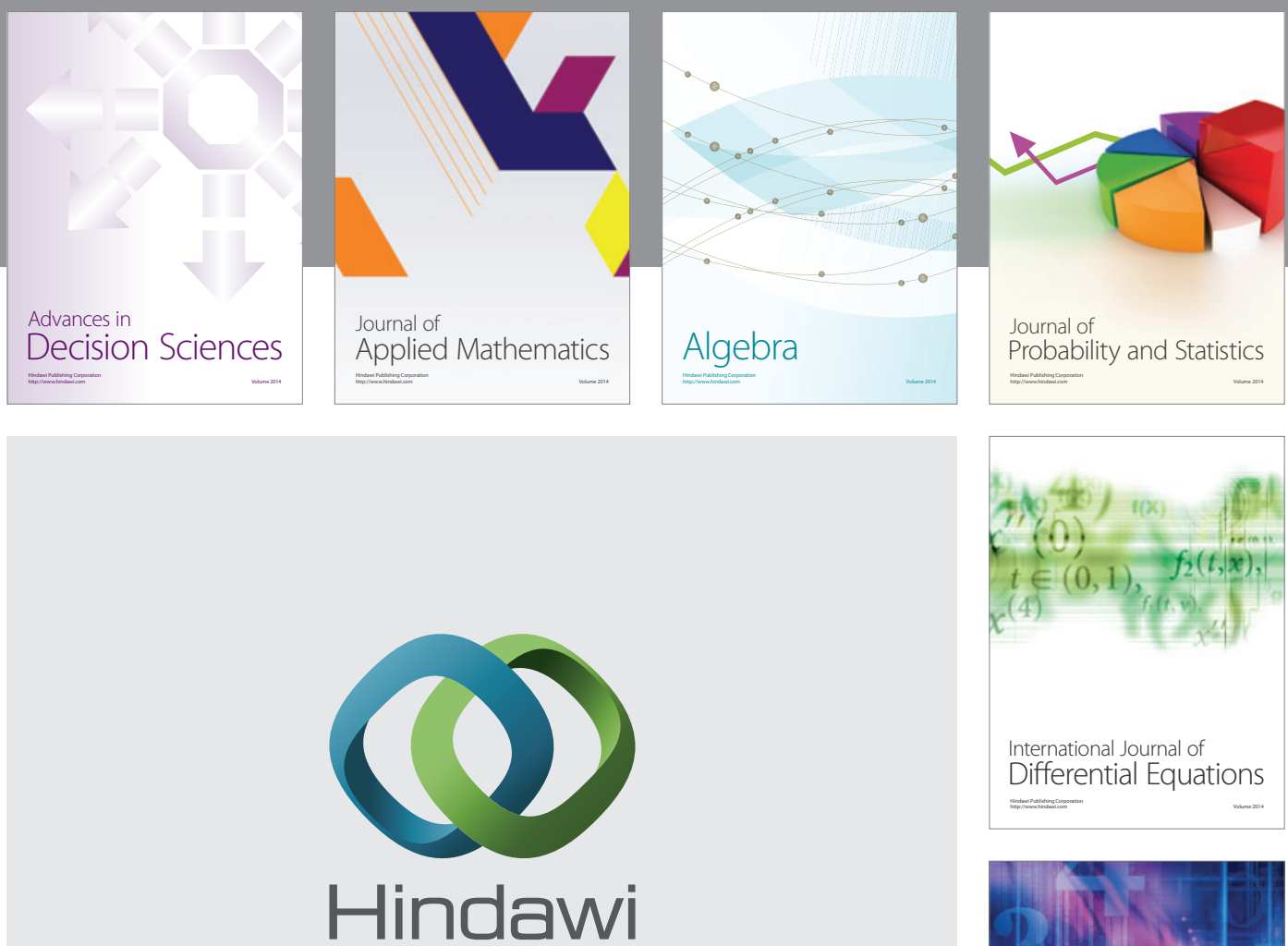

Submit your manuscripts at http://www.hindawi.com
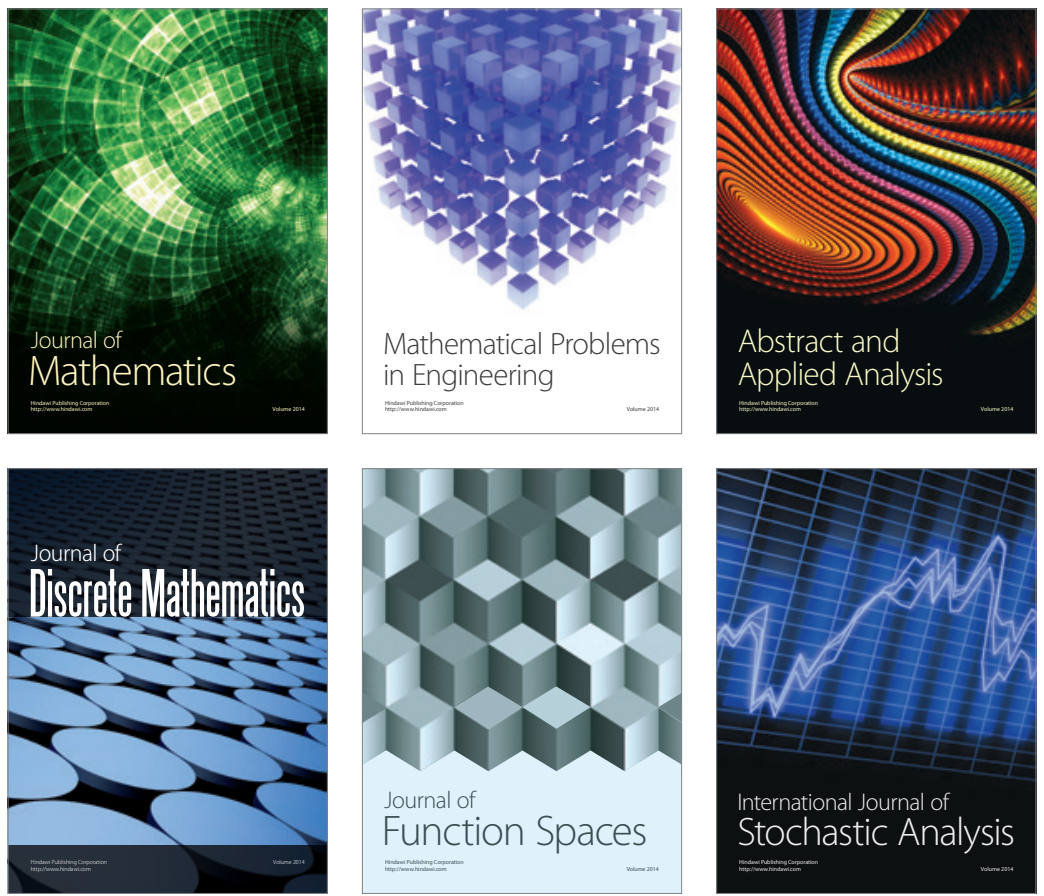

Journal of

Function Spaces

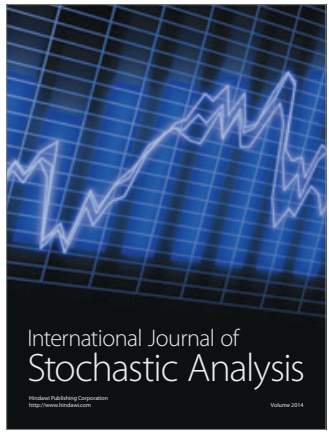

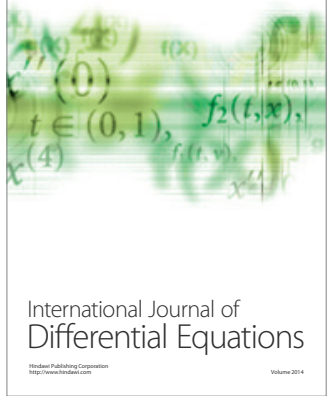
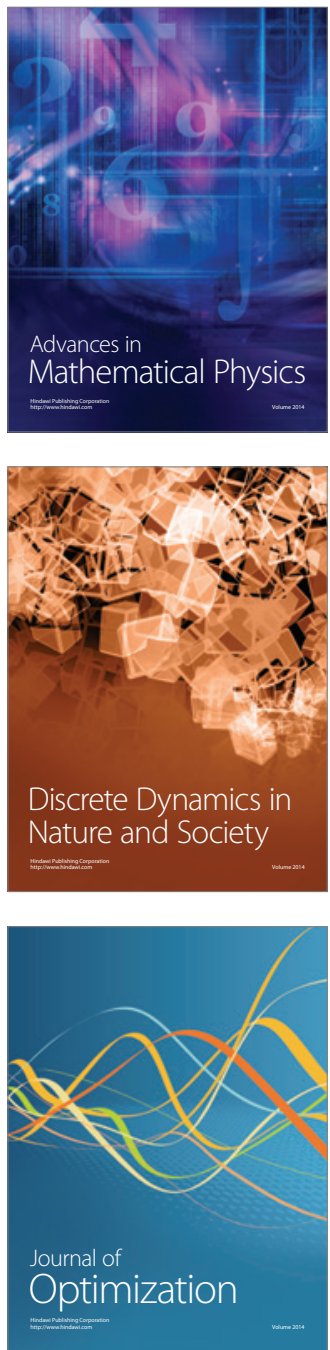